\title{
Multifunctional Nanotheranostics: Do We Really Need Them?
}

\section{Jagat R. Kanwar}

Laboratory of Immunology and Molecular Biomedical Research, Centre for Biotechnology and Interdisciplinary Biosciences, Institute for Technology and Research Innovation, Deakin University, Geelong, Australia

"There's plenty of room at the bottom", is one of the visionary concepts pertaining to molecular nanotechnology put forward by the nobel laureate Richard Feynman, envisaging the fabrication of exotic multifunctional nanoscale therapeutic platforms and medical devices that lead to genesis of "Nanomedicine" [1]. In turn, consistent effort from various scientific disciplines including material sciences, biotechnology, medicine and computational sciences have revolutionized the area of nanomedicine leading to exquisite edification of existing nano therapeutics into a new generation platform called "multifunctional nanotheranostics" $[1,2]$. Multifunctional nanotheranostic platforms preferentially achieve contemporaneous cancer diagnosis, target specific drug delivery and therapeutic imaging by employing myriad of ultra modern combinatorial technologies including RNA interference, aptamers, locked nucleic acid conjugates (LNA), fluorescent imaging, magnetic resonance imaging (MRI), positron emission tomography (PET), single photon emission computed tomography (SPECT), photodynamic laser therapy (PDT), magnetic therapy [3,4]. It would certainly be an understatement to undermine the devastation triggered in human cancers out bounding conventional chemo and radio therapeutics [5]. Nevertheless, target specific drug delivery is profoundly comphrended by the ever acclimatizing and evolving complexation in the functional modulation of nodal cancer cell signalling networks [6]. However, at this point nanotheranostics can sneak through in the current therapeutic basket to retaliate the battle with critical angiogenic signalling modules of cancer cells [2]. Notable demarcation of cancerous from normal tissues including increased angiogenesis, leaky blood vasculature, expression of tumour specific biomarkers.etc can be potentially exploited to empower and ship the nanotheranostics through the human body to selectively deliver anticancer agents to infected sites relatively leaving normal tissues unharmed $[7,8]$. With the advent of major toxicology associated with current treatment regimes, a new class of safe and natural product derived therapeutics including lactoferrin, curcumin, neem etc are currently in lime light of research focus for their outstanding functional ability in abrogating key nodal cancer signalling paradigms thereby escalating remarkable tumour growth inhibition $[9,10]$. A predominant class of natural product based super paramagnetic iron oxide nanoparticle (SPIONS) nanotheranostic agents inconjucture with tumour specific targeting entities including epithelial cell adhesion molecule (EPCAM), aptamer variants in combination with locked nucleic acid conjugates including LNA - nucleolin aptamer, LNA - EPCAM aptamer were fabricated $[3,11]$. The above mentioned nanotheranostic agents were able to selectively induce tumour apoptosis exhibiting distinct anti tumour activity as envisaged by advanced fluorescence imaging techniques [11]. Furthermore, theranostic investigations by utilisation of plethora of ultra modern imaging techniques including MRI, PET, SPECT, PDT are into consideration [3].

In conclusion, multifunctional nanotheranostics in partnership with various upcoming technologies including bioinformatics, systems biology will certainly hold substantial promise in opening new vistas for effective management of clinical cancers by their multimodal action $[1,12]$.

\section{References}

1. Freitas RA Jr (2005) What is nanomedicine? Nanomedicine 1: 2-9.

2. Chen X, Gambhir SS, Cheon J (2011) Theranostic nanomedicine. Acc Chem Res 44: 841

3. Kanwar JR, Kamalapuram SK, Kanwar RK (2012) Survivin Signaling in Clinical Oncology: A Multifaceted Dragon. Med Res Rev.

4. Kievit FM, Zhang M (2011) Cancer nanotheranostics: improving imaging and therapy by targeted delivery across biological barriers. Adv Mater 23 $\mathrm{H} 217-\mathrm{H} 247$.

5. Kanwar JR, Kamalapuram SK, Kanwar RK (2011) Targeting survivin in cancer: the cell-signalling perspective. Drug Discov Today 16: 485-494.

6. Kanwar RK, Cheung CH, Chang JY, Kanwar JR (2010) Recent advances in anti-survivin treatments for cancer. Curr Med Chem 17: 1509-1515.

7. Kanwar JR, Kanwar RK, Mahidhara G, Cheung CH (2012) Cancer Targeted Nanoparticles Specifically Induce Apoptosis in Cancer Cells and Spare Normal Cells. Aust J Chem 65: 5-14

8. Kanwar JR, Mahidhara G, Kanwar RK (2011) Antiangiogenic therapy using nanotechnological-based delivery system. Drug Discov Today 16: 188-202.

9. Kanwar JR, Kamalapuram SK, Kanwar RK (2010) Targeting survivin in cancer: patent review. Expert Opin Ther Pat 20: 1723-1737.

10. Kanwar JR, Palmano KP, Sun X, Kanwar RK, Gupta R, et al. (2008) 'Ironsaturated' lactoferrin is a potent natural adjuvant for augmenting cancer chemotherapy. Immunol Cell Biol 86: 277-288.

11. Kanwar JR, Roy K, Kanwar RK (2011) Chimeric aptamers in cancer celltargeted drug delivery. Crit Rev Biochem Mol Biol 46: 459-477.

12. Kreeger PK, Lauffenburger DA (2010) Cancer systems biology: a network modeling perspective. Carcinogenesis 31: 2-8

${ }^{*}$ Corresponding author: Dr. Jagat R. Kanwar, PhD, Associate Professor of Immunology and Cell Biology, Laboratory of Immunology and Molecular Biomedica Research, Centre for Biotechnology and Interdisciplinary Biosciences, Institute for Technology and Research Innovation, Deakin University, Geelong, Australia, Tel: 00613 52271148; Fax: 00613 52272539; E-mail: Jagat.Kanwar@deakin.edu.au

Received October 04, 2012; Accepted October 08, 2012; Published October 10, 2012

Citation: Kanwar JR (2012) Multifunctional Nanotheranostics: Do We Really Need Them? J Cancer Sci Ther 4: xxii-xxii. doi:10.4172/1948-5956.1000e114

Copyright: (c) 2012 Kanwar JR. This is an open-access article distributed unde the terms of the Creative Commons Attribution License, which permits unrestricted use, distribution, and reproduction in any medium, provided the original author and source are credited. 